Article

\title{
The Effect of Zoledronic Acid on Serum Biomarkers among Patients with Chronic Low Back Pain and Modic Changes in Lumbar Magnetic Resonance Imaging
}

\author{
Katri Koivisto ${ }^{1, *}$, Jaro Karppinen 1,2,3 ${ }^{(D}$, Marianne Haapea 1,2,4, Jyri Järvinen 1,4, Eero Kyllönen ${ }^{1}$, \\ Osmo Tervonen ${ }^{4}$, Jaakko Niinimäki ${ }^{4}$, Mauro Alini ${ }^{5}$, Jeffrey Lotz ${ }^{6}$, Stefan Dudli ${ }^{7}$, \\ Dino Samartzis ${ }^{8}$, Juha Risteli ${ }^{9}$, Marja-Leena Majuri ${ }^{1}$, Harri Alenius ${ }^{10}$ and Sibylle Grad ${ }^{5}$ \\ 1 Medical Research Center Oulu, Oulu University Hospital and University of Oulu, 90220 Oulu, Finland; \\ jaro.karppinen@ttl.fi (J.K.); marianne.haapea@oulu.fi (M.H.); jyri.jarvinen@ppshp.fi (J.J.); \\ eero.kyllonen@oulu.fi (E.K.); marjaleena.majuri@gmail.com (M.-L.M.) \\ 2 Center for Life Course Health Research, University of Oulu, 90220 Oulu, Finland \\ 3 Finnish Institute of Occupational Health, 90220 Oulu, Finland \\ 4 Research Unit of Medical Imaging, Physics and Technology, University of Oulu, 90220 Oulu, Finland; \\ osmo.tervonen@oulu.fi (O.T.); jaakko.niinimaki@oulu.fi (J.N.) \\ 5 AO Research Institute Davos, 7270 Davos Platz, Switzerland; mauro.alini@aofoundation.org (M.A.); \\ sibylle.grad@aofoundation.org (S.G.) \\ 6 Department of Orthopaedic Surgery, University of California San Francisco, San Francisco, CA 94143, USA; \\ jeffrey.lotz@ucsf.edu \\ 7 Center of Experimental Rheumatology, University Hospital Zurich, 8008 Zurich, Switzerland; \\ stefan.dudli@usz.ch \\ 8 Department of Orthopedic Surgery, RUSH University, Chicago, IL 60612, USA; Dino_Samartzis@rush.edu \\ 9 Northern Finland Laboratory Centre NordLab, Oulu University Hospital, 90220 Oulu, Finland; \\ juha.risteli@oulu.fi \\ 10 Institute of Environmental Medicine, Karolinska Institute, 17177 Stockholm, Sweden; harri.alenius@ki.se \\ * Correspondence: ka.koivisto@elisanet.fi
}

Received: 16 October 2019; Accepted: 2 December 2019; Published: 4 December 2019

\begin{abstract}
The aim of the current study was to compare changes in serum biomarkers, including inflammatory mediators, signaling molecules, growth factors and markers of bone turnover after a single intravenous infusion of $5 \mathrm{mg}$ zoledronic acid (ZA, a long-acting bisphosphonate; $n=20$ ) or placebo $(n=20)$ among patients with Modic changes $(\mathrm{MC})$ and chronic low back pain in a randomized controlled design. The MCs were classified into M1, predominating M1, predominating M2, and M2. We measured the serum concentrations of 39 biomarkers at baseline, and one month and one year after treatment. After Benjamini-Hochberg (B-H) correction, we observed significant differences in three biomarkers over one year: Interferon- $\gamma$-inducible protein (IP-10) had risen in the ZA group $(p=0.005)$, whereas alkaline phosphatase (AFOS) and intact procollagen I N-terminal propeptide (iPINP) had significantly decreased in the ZA group, but had not changed in the placebo group $(p<0.001$ for both). Change in iPINP correlated with change in the volume of all MC and M1 lesions. ZA downregulated bone turnover markers as expected and, surprisingly, increased the chemokine IP-10 relative to placebo treatment. This adds to our knowledge of the effects of ZA on MC and the biomarkers that signal this process.
\end{abstract}

Keywords: serum biomarkers; magnetic resonance imaging; Modic change; randomized trial; zoledronic acid; chronic low back pain 


\section{Introduction}

A Modic change (MC) is a vertebral bone marrow change visible in magnetic resonance imaging (MRI). Three different types of MC have been described, of which Type 1 (M1) shows fibrovascular replacement of the bone marrow and represents an inflammatory lesion; Type 2 MC (M2) shows fatty replacement of the red bone marrow; and Type $3 \mathrm{MC}$ (M3) demonstrates subchondral bone sclerosis [1-4]. MC is associated with chronic low back pain (CLBP) [5-7] and M1 shows a stronger association with CLBP than other MC types [8-10]. In a Finnish patient study, conversion from M1 to M2 over two years was associated with improvement of pain symptoms [11]. Similar findings have been obtained among Danish patients [12].

Both infectious or autoimmune etiologies have been suggested as causes of $\mathrm{MC}$, and both presuppose structural damage of the endplate [13-15]. However, improved diagnostic tools are needed to detect early MC lesions. One novel pre-clinical technique that holds great clinical potential is nuclear magnetic resonance spectroscopy, which is a noninvasive method to quantify Cutibacterium (formerly Propionibacterium) acnes load on the intervertebral discs [16,17]. There is also an urgent need to develop other MC biomarkers in order to increase early diagnosis and the choice of the right treatment and establishment of MC prognosis [13].

A biomarker is defined as a characteristic that is objectively measured and evaluated as an indicator of normal biologic or pathogenic processes, or pharmacologic responses to a therapeutic intervention [18]. Molecular diagnostic techniques have developed in recent years and may be helpful in revealing diseases at their early stage. However, only two studies of MC serum biomarkers have been published so far. In the first, serum high-sensitive C-reactive protein (hs-CRP) was higher among patients with M1 than among those with M2, or those without MC but with CLBP [19]. Another small French case-control study in turn found no difference in bone mineral density and bone remodeling marker levels (serum C-terminal crosslinked type 1 telopeptide, procollagen type $1 \mathrm{~N}$-terminal propeptide, osteocalcin, and bone-specific alkaline phosphatase) among chronic LBP patients with M1 or without MC [20]. We have previously demonstrated how a single intravenous infusion of $5 \mathrm{mg}$ zoledronic acid (ZA) decreased the intensity of pain among patients with CLBP in the short-term in comparison to placebo [21]. We hypothesized that the beneficial effect of ZA on symptoms may be partly due to the reduction of inflammation, which in turn may be reflected by serum biomarkers related to inflammation and bone turnover. Therefore, the objective of our study was to compare the effect of a single intravenous infusion of $5 \mathrm{mg}$ ZA to that of placebo infusion on the change in the serum biomarker profile of patients with $\mathrm{MC}$, and to evaluate whether serum biomarkers correlate with the type and size of MC at baseline.

\section{Materials and Methods}

\subsection{Study Design and Selection of Patients}

The study population consisted of patients with CLBP and MC in lumbar MRI [21]. Inclusion criteria were LBP for at least three months, LBP intensity of at least six on the 10-cm Visual Analog Scale (VAS) or an Oswestry Disability Index (ODI) [22] of at least 30\% and an MC (M1, M2, or M1/2) in MRI [21].

The Oulu University Hospital ethics committee approved the study protocol (121/2008, 18 August 2008). Informed consent was obtained from all participants (ClinicalTrials.gov NCT01330238).

\subsection{Treatment Intervention}

Patients were randomized into a ZA group with an infusion of $5 \mathrm{mg}$ of ZA $(n=20)$ or a placebo group (saline infusion, $n=20$ ). Before the administration of the infusion, all the patients received 600 $\mathrm{mg}$ of ibuprofen or $1 \mathrm{~g}$ of paracetamol orally to prevent potential acute phase reactions, and 100,000 units of Vitamin D (VigantolR) orally to avoid hypocalcemia [21]. 


\subsection{Magnetic Resonance Imaging}

The average interval between baseline imaging and infusion was four months (range 0.4 to 11.5 months). Follow-up imaging was performed on average 11.9 months (range 11 to 13 months) after infusion [23]. The MRI equipment of the baseline imaging included five 1.5 T units (GE Signa Twinspeed, General Electric Medical Systems, Milwaukee, WI, USA; Philips Achieva and Philips Intera, Philips Medical Systems, Eindhoven, The Netherlands; Siemens Avanto and Siemens Espree, Siemens Medical, Erlangen, Germany), a 0.34 T unit (Siemens Magnetom C, Siemens Medical, Erlangen, Germany) and a 0.23 T unit (Philips Panorama, Philips Medical Systems, Eindhoven, The Netherlands). The imaging sequences were sagittal T1-weighted (T1 W) turbo spin-echo (TSE) or fast spin-echo (FSE) with fluid attenuation inversion recovery (FLAIR), sagittal T2-weighted (T2 W) TSE/FSE, and short tau inversion recovery sequences (STIR). The specific imaging parameters have been described previously [23]. The MRI equipment of the one-year follow-up imaging included two 1.5 T units (GE Signa Twinspeed and GE Optima, General Electric Medical Systems, Milwaukee, WI, USA) and a 3 T unit (Siemens Skyra, Siemens Medical, Erlangen, Germany). Imaging protocols were established for clinical spine imaging.

\subsection{Image Analysis}

We analyzed the MRIs for type and volume of each MC from sagittal images at a clinical workstation (Neaview Radiology, version 2.23, Neagen Corporation, Oulu, Finland) [23]. MC type was assessed using T1 $\mathrm{W}$ and $\mathrm{T} 2 \mathrm{~W}$ images as previously described [10]. Four MC type groups were created: M1 (100\%), predominating M1 (M1/2 (65:35\%)), predominating M2 (M1/2 (35:65\%)) and M2 (100\%). The first two groups were considered M1-dominant, and the latter two M2-dominant. M1 was defined as consisting of purely oedemic signal changes and M2 consisting of purely fatty signal changes. Predominating M1 was defined as mixed Type 1/2 MC with more oedemic signal changes and predominating M2 as Type $1 / 2 \mathrm{MC}$ with more fatty signal changes. The MC area $\left(\mathrm{cm}^{2}\right)$ was determined from $\mathrm{T} 2 \mathrm{~W}$ images. The volume $\left(\mathrm{cm}^{3}\right)$ of each MC lesion was calculated by multiplying the area with the spacing.

\subsection{Analysis of Serum Biomarkers}

Whole blood was drawn at noon from fasting patients using serum collection tubes prior to the intervention (baseline), at one-month and at one-year follow-up. We told all the participants not to exercise, eat, or drink the previous evening or the morning before the blood were to be taken. The blood samples were allowed to clot at room temperature for 30-60 min and then centrifuged at $2500 \times g$ for $10 \mathrm{~min}$ at $4{ }^{\circ} \mathrm{C}$. The resulting serum supernatant was stored in aliquots, first at $-20^{\circ} \mathrm{C}$, and were then transferred within $2-20 \mathrm{~h}$ to $-70{ }^{\circ} \mathrm{C}$. We took serum samples from 40 patients at three timepoints $(n=120)$ to assess the selected inflammatory and signaling molecules, growth factors and markers of bone turnover. We measured the serum concentrations of 50 biomarkers, and quantified 38 biomarkers using electro-chemiluminescent based multi-array immunoassays from MesoScale Discovery (MSD, Rockville, MD, USA), following the protocol of the manufacturer: Chemokine panel: Eotaxin, eotaxin-3, interferon-gamma-inducible protein (IP)-10, macrophage inflammatory protein (MIP)-1-alpha, MIP-1-beta, monocyte chemotactic protein (MCP)-1, MCP-4, macrophage-derived chemokine (MDC)-1, regulated on activation normal T-expressed and secreted (RANTES), thymus and activation regulated chemokine (TARC); Cytokine panel: Granulocyte-macrophage colony stimulating factor (GM-CSF), interleukin (IL)-5, interleukin (IL)-7, IL-12/23p40, IL-15, IL-16, IL-17A, tumor necrosis factor (TNF)-beta; Inflammation panel: Interferon (IFN)-gamma, IL-1-alpha, IL-1-beta, IL-2, IL-6, IL-8, IL-12p70, TNF-alpha, IL-4, IL-10, IL-13; Angiogenesis panel: Vascular endothelial growth factor (VEGF)-A, VEGF-C, VEGF-D, TEK receptor tyrosine kinase (Tie-2), fms related tyrosine kinase 1 (Flt-1), basic fibroblast growth factor (bFGF); and Vascular injury panel: Intercellular adhesion molecule (ICAM)-1, vascular cell adhesion molecule (VCAM)-1, serum amyloid A (SAA). To analyze the interleukin 1 receptor antagonist (IL-1RA), we used epidermal growth factor (EGF), hepatocyte growth 
factor (HGF), interferon-alpha (IFN-A), interleukin-2 receptor (IL-2R) and monokine induced by gamma-interferon (MIG-1), a Cytokine Human Magnetic Multi-Plex Panel (Thermo Fisher Scientific, Waltham, MA, USA), according to the manufacturer's instructions. The receptor activator of NF- $\mathrm{kB}$ ligand (RANKL) was measured using a commercial Luminex single plex kit (Human RANKL single plex kit, MilliplexTM Map, Merck Millipore, Billerica, MA, USA), and soluble interleukin-1 receptor, type II (IL-1sRII) with a human IL-1RII Quantikine Elisa kit (R\&D Systems, Minneapolis, MN, USA). All Luminex assays were performed using Luminex xMAP Technology (Bio-Plex 200 System; BioRad, Hercules, CA, USA). For the IL-1sRII Elisa assay, absorption was read at $450 \mathrm{~nm}$ using an ELISA plate absorbance reader, and the correction was made at $570 \mathrm{~nm}$ (Multiskan MS, Thermo Scientific, Vantaa, Finland). Plasma alkaline phosphatase activity (AFOS) was analyzed using the IFCC recommended enzymatic method (Advia, Siemens, Germany). Serum highly sensitive CRP (hs-CRP) was analyzed using a nephelometric instrument (BN ProSpec, Siemens, Germany). Intact procollagen I N-terminal propeptide (iPINP) and C telopeptide of type I collagen (CTX-I) analyses were performed using the IDS-iSYS Multi-Discipline Automated System (IDS, Bolton, UK). As the results of 11 biomarkers (EGF, GM-CSF, IL-1A, IL-1B, IL-1RA, IL-2, IL-4, IL-5, IL-10, IL-12p70, IL-13) were below the limit of quantification for more than half of the samples in the whole study, we excluded them from the final results.

\subsection{Statistical Analyses}

The background variables are presented as frequencies with proportions and means with standard deviations. We compared these variables of the ZA and placebo groups using the chi-square test or independent samples $t$-test, respectively. The serum biomarkers at baseline, one month and one year are separately presented as medians with interquartile range for the ZA and placebo groups. The change in serum biomarkers from baseline to one month and one year in the ZA and placebo groups, and in the M1- and M2-dominant MC were compared using Mann-Whitney's U-test. The change in the serum biomarkers from baseline to one month and one year in the ZA and placebo groups, and in the M1- and M2-dominant MC were compared separately, using the Wilcoxon signed-rank test. We analyzed the correlation of the change in serum biomarkers from baseline to one year with a change in primary $\mathrm{MC}$ total volume, M1 volume and M2 volume, and with a change in LBP using Spearman's rank correlation coefficient. The Benjamini-Hochberg (B-H) procedure, with a 10\% false discovery rate, was used to correct for multiple comparisons. We used IBM SPSS Statistics, version 24.0 to conduct the analyses.

\section{Results}

\subsection{Study Population}

All 40 enrolled, eligible patients participated in the one-year follow-up. There were no statistically significant differences between the baseline demographic characteristics of the ZA and placebo groups (Table 1). The patients' mean age was 50 (SD 8.3) and mean body mass index (BMI) 26.8 (SD 3.2). The median duration of LBP was 330 (IQR 216-365) days and the mean VAS score for LBP was 6.7 (SD 1.5). The baseline serum biomarker concentrations of the placebo and ZA groups did not differ significantly.

The results of 11 biomarkers were below the limit of quantification for more than half of the samples in the whole study and were thus excluded from the final results. Table 2 presents the results of all the 39 serum biomarkers analyzed. The significant changes are in bold in Table 2 and the markers that remained significant after B-H correction are marked with an asterisk. At one-month follow-up, IL-8 in the placebo group and intact procollagen I N-terminal propeptide (iPINP) in the ZA group had decreased, whereas IP-10, MCP-1, SAA, and hs-CRP had increased in the ZA group (Table 2, Figures S1-S4). Compared to placebo, the decrease in biomarker concentration from baseline to one month was greater in the ZA group in terms of iPINP (median of change $-9.3 \mathrm{vs}$. $-0.7 \mathrm{ng} / \mathrm{mL}, p=0.018$ ) and in CTX-1 $(-0.27$ vs. $-0.02 \mathrm{pg} / \mathrm{mL}, p=0.009)$. At one month, there were no significant differences between the $\mathrm{ZA}$ and placebo groups after $\mathrm{B}-\mathrm{H}$ correction. 
Table 1. Characteristics of patients treated with zoledronic acid (ZA) and placebo. Group differences between age, body mass index (BMI), and Modic changes (MC) volumes were analyzed using the $t$-test. Otherwise, the chi-square test was used.

\begin{tabular}{|c|c|c|c|}
\hline Variable & ZA & Placebo & $p$-Value \\
\hline Age (years), mean (SD) & $49.2(9.3)$ & $51.5(7.3)$ & 0.400 \\
\hline BMI $\left(\mathrm{kg} / \mathrm{m}^{2}\right)$, mean (SD) & $26.1(3.3)$ & $27.4(3.2)$ & 0.203 \\
\hline Males, $n(\%)$ & $15(75.0 \%)$ & $11(55.0 \%)$ & 0.320 \\
\hline Regular smoker, $n(\%)$ & $5(25.0 \%)$ & $6(30.0 \%)$ & $>0.999$ \\
\hline Primary MC type, $n(\%)$ & & & 0.103 \\
\hline MI & $3(15.0 \%)$ & $3(15.0 \%)$ & \\
\hline $\mathrm{MI} / \mathrm{II}-1$ & $14(70.0 \%)$ & $7(35.0 \%)$ & \\
\hline $\mathrm{MI} / \mathrm{II}-2$ & $2(10.0 \%)$ & $8(40.0 \%)$ & \\
\hline MII & $1(5.0 \%)$ & $2(10.0 \%)$ & \\
\hline Number of MCs, $n(\%)$ & & & 0.333 \\
\hline 1 & $10(50.0 \%)$ & $14(70.0 \%)$ & \\
\hline 2 or more & $10(50 \%)$ & $6(30.0 \%)$ & \\
\hline \multicolumn{4}{|l|}{ Volume of primary MC, mean (SD) } \\
\hline Type 1 & $7443(4474)$ & $5044(3551)$ & 0.068 \\
\hline Type 2 & $4542(3897)$ & $5868(4840)$ & 0.346 \\
\hline Total & $11,985(5140)$ & $10,911(5963)$ & 0.546 \\
\hline Osteoarthritis, $n(\%)$ & $1(5.0 \%)$ & $6(30.0 \%)$ & 0.091 \\
\hline Back pain (VAS), mean (SD) & $6.6(1.4)$ & $6.8(1.6)$ & 0.646 \\
\hline Leg pain (VAS), mean (SD) & $3.0(3.1)$ & $2.9(2.3)$ & 0.864 \\
\hline Oswestry disability index, mean (SD) & $30.1(11.0)$ & $34.9(9.8)$ & 0.157 \\
\hline
\end{tabular}

Table 2. Median concentrations and interquartile range (IQR) of serum biomarkers at baseline, one month and one year according to intervention (zoledronic acid (ZA) or placebo infusion). $\mathrm{P}^{1}$ shows the significance of the change in each respective biomarker concentration from baseline to one month or from baseline to one year. $\mathrm{P}^{2}$ shows the significance of the difference in change from baseline to one month or from baseline to one year in the concentration of each biomarker between the ZA and placebo groups. Significant $p$-values are in bold.

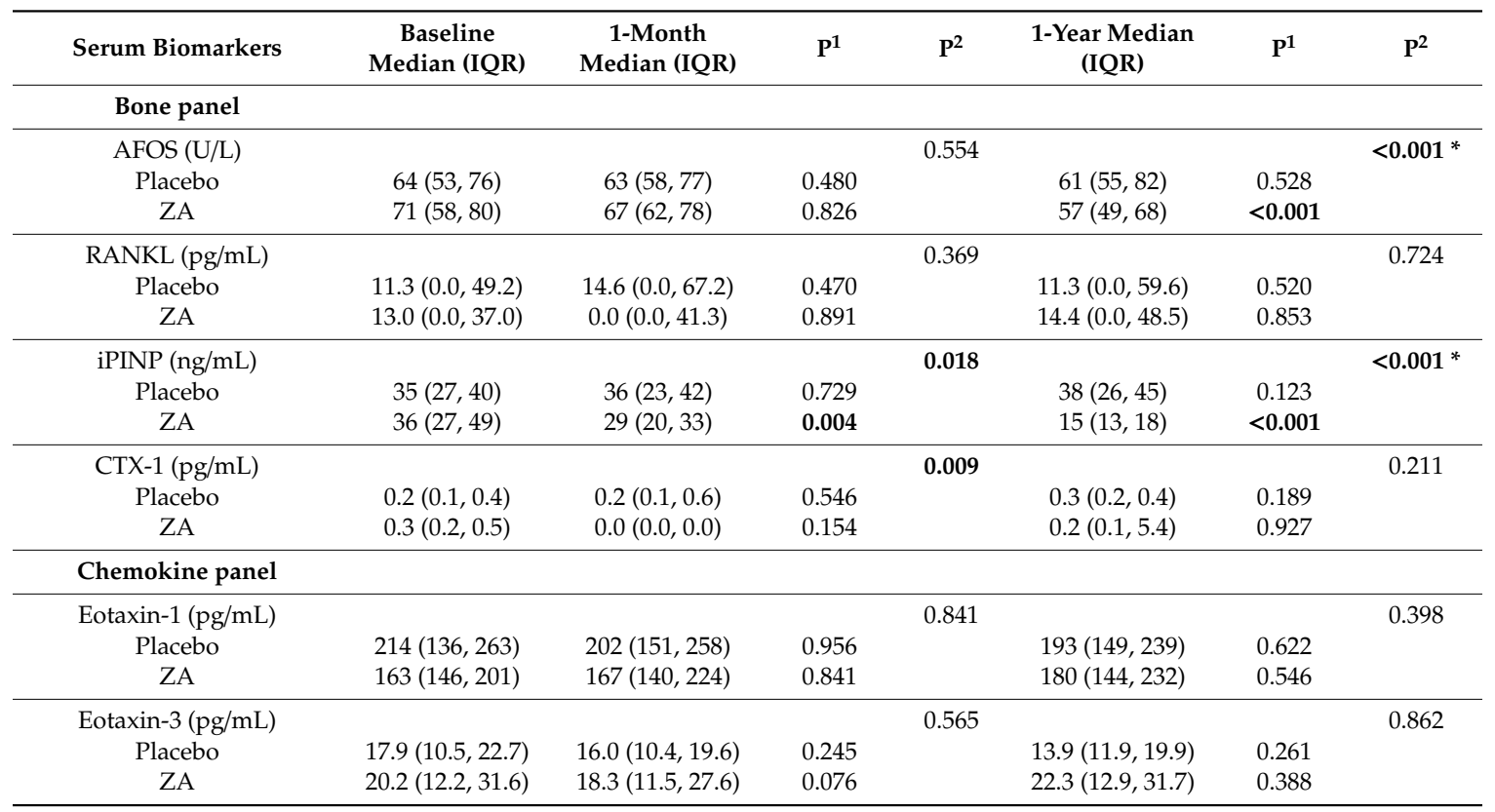


Table 2. Cont

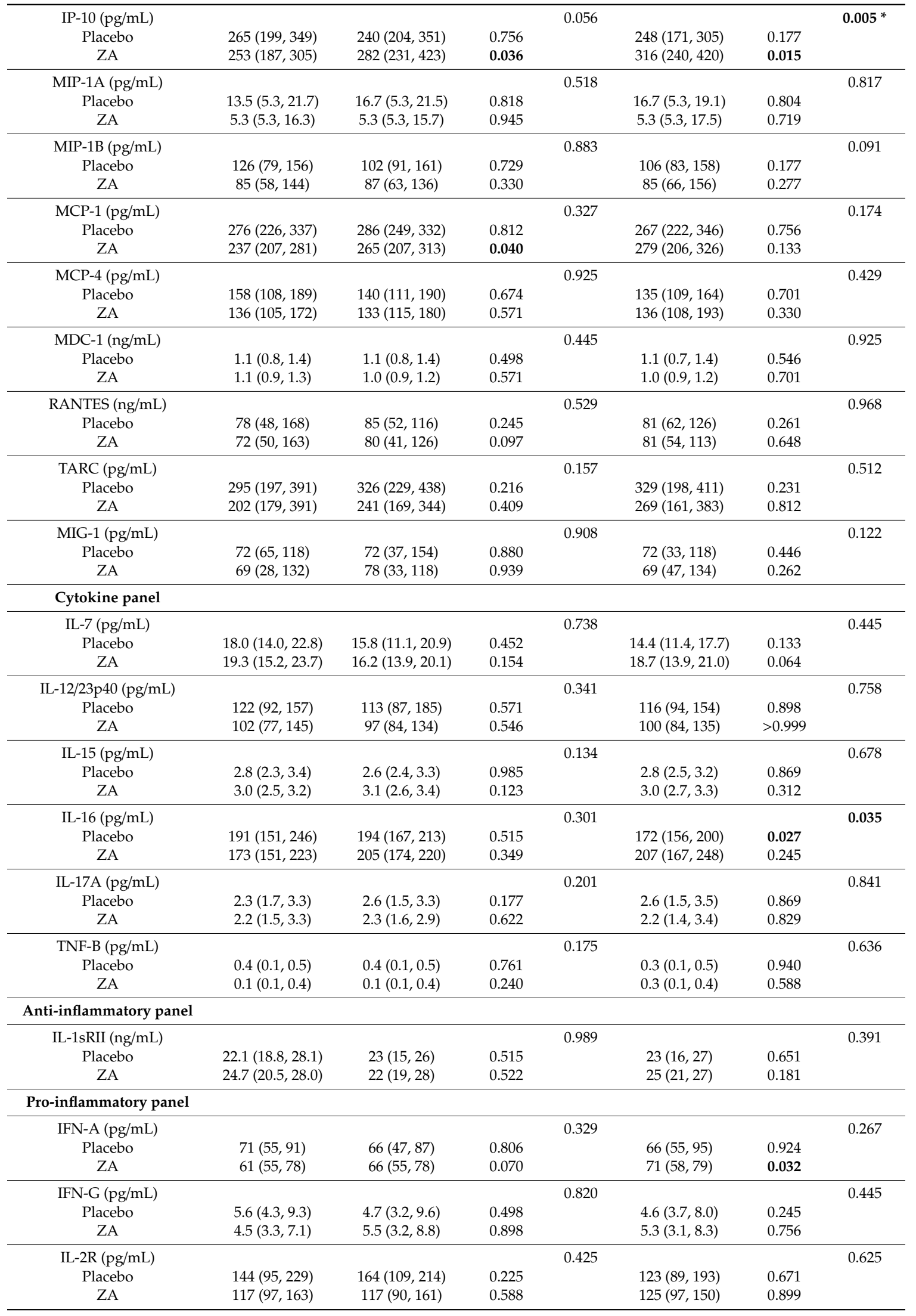


Table 2. Cont.

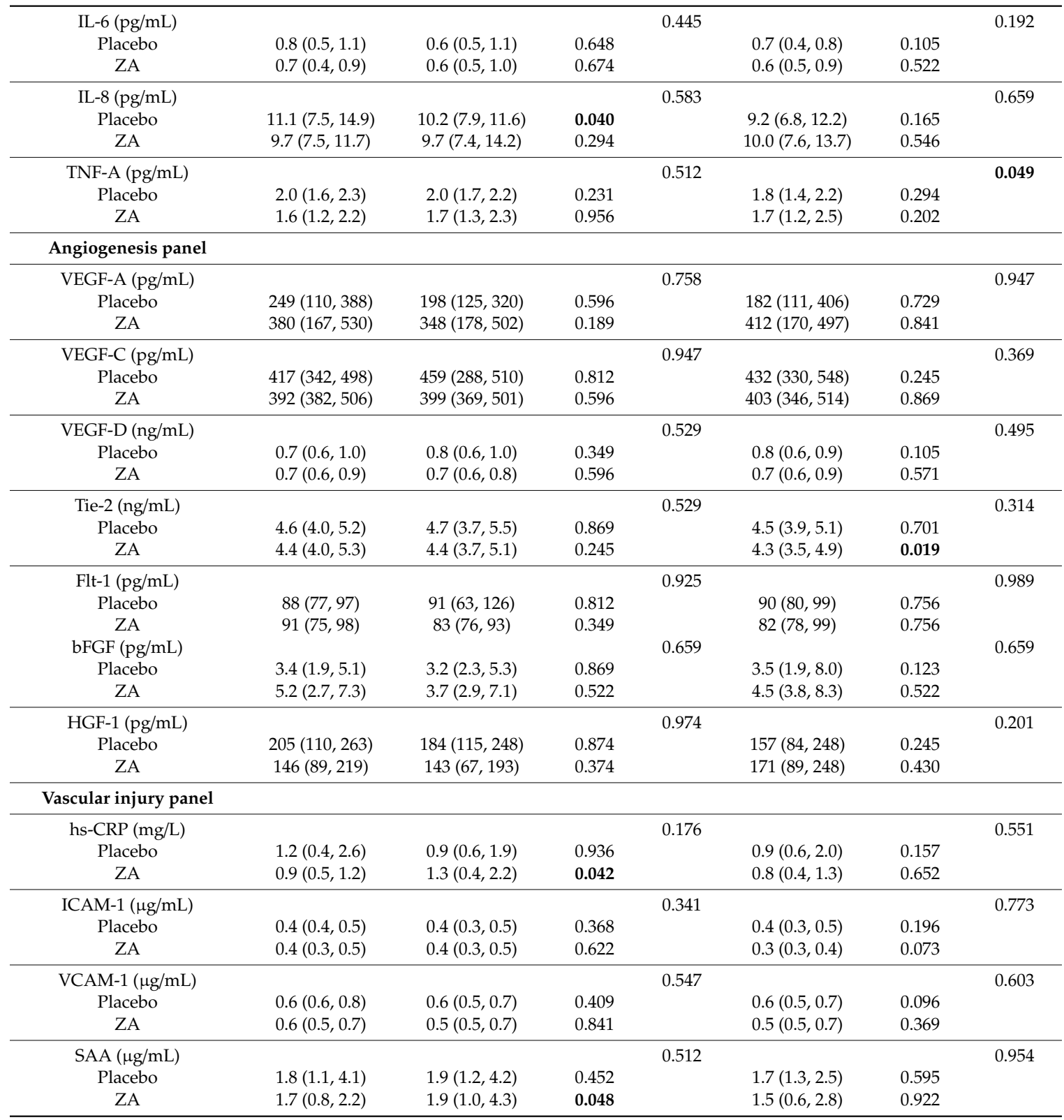

${ }^{1}$ Significance in Wilcoxon signed rank test, ${ }^{2}$ Significance in Mann-Whitney's test; ${ }^{*}$ significant after B-H correction; AFOS $=$ alkaline phosphatase; $\mathrm{bFGF}=$ basic fibroblast growth factor beta; $\mathrm{CTX} 1=\mathrm{C}$ telopeptide of type I collagen; $\mathrm{Flt}=\mathrm{fms}$ related tyrosine kinase $(=\mathrm{VEGFR} 1) ; \mathrm{HGF}=$ hepatocyte growth factor; hs-CRP $=$ high-sensitive C-reactive protein; ICAM = intercellular adhesion molecule; IL = interleukin; IFN = interferon; IP = interferon- $\gamma$-inducible protein; iPINP = intact procollagen I $\mathrm{N}$-terminal propeptide; $\mathrm{MCP}=$ monocyte chemotactic protein; $\mathrm{MDC}=$ macrophage derived chemokine; $\mathrm{MIG}=$ monokine induced by gamma-interferon; $\mathrm{MIP}=$ macrophage inflammatory protein; $\mathrm{RANKL}=$ receptor activator of NF-KB ligand; RANTES = regulated upon activation, normally T-expressed, and presumably secreted; $\mathrm{SAA}=$ serum amyloid $\mathrm{A} ; \mathrm{TARC}=$ thymus and activation-regulated chemokine; Tie $=$ TEK receptor tyrosine kinase; $\mathrm{TNF}=$ tumor necrosis factor; VCAM $=$ vascular cell adhesion molecule; VEGF $=$ vascular endothelial growth factor.

At one-year follow-up, IL-16 in the placebo group, and Tie-2, AFOS and iPINP in the ZA group had decreased, whereas IP-10 and IFN-A had increased in the ZA group. During the one-year follow-up, one cytokine biomarker (IL-16) had decreased in the placebo group and two biomarkers (IP-10 and IFN-A) had increased in the ZA group. The change in IP-10 (decrease of -13.5 in the placebo group vs. increase of $61.1 \mathrm{pg} / \mathrm{mL}$ in the ZA group, $p=0.005$ ) was significant after $\mathrm{B}-\mathrm{H}$ correction. Two serum biomarkers (AFOS and iPINP) had not changed in the placebo group but had decreased in the ZA group ( 2.5 vs. $-9.0 \mathrm{U} / \mathrm{l}, p<0.001$ and 1.0 vs. $-19.1 \mathrm{ng} / \mathrm{mL}, p<0.001$; respectively). At one year, 
two biomarkers had significantly decreased in the ZA group after B-H correction: AFOS (71 to 57, $p<0.001$ ) and iPINP (36 to $15, p<0.001$; Table 2).

Among the patients with M2 at baseline, TARC had increased significantly during the one-year follow-up, whereas among the patients with M1 at baseline, AFOS and iPINP had decreased significantly (Table S1). In the ZA group, the changes in total volume of MC and M1 volume during the one-year follow-up correlated positively with the changes in iPINP (Spearman's correlations (rho) 0.65 and 0.49). None of the other changes were significant and are presented in Table S2. The changes in LBP intensity did not correlate statistically significantly with the changes in the concentration of any biomarker, as shown in Table S3.

\section{Discussion}

In the present study, a single intravenous infusion of $5 \mathrm{mg}$ ZA gave rise to different trends in the $\mathrm{ZA}$ and placebo groups, leading to significant differences at one-year follow-up in one chemokine (IP-10), which had increased in the ZA group and decreased in the placebo group, whereas two bone metabolism biomarkers (AFOS and iPINP) had decreased in the ZA group. Change in iPINP correlated with the change in the volume of all MC and M1 lesions.

We measured serum levels of AFOS, iPINP, and CTX-1 to evaluate bone formation and resorption. Of the bone biomarkers, iPINP decreased significantly at both one month and one year and AFOS at one year in the ZA group. The decreased concentrations in the bone biomarkers were not unexpected, as ZA has shown to suppress bone remodeling among post-menopausal women with osteoporosis [24,25]. The former [24] documented a 58\% reduction in iPINP at one year after ZA administration among postmenopausal women with osteoporosis, whereas in the present study, iPINP concentration decreased by $19.4 \%$ at one month and $58.3 \%$ at one year, compared with $2.8 \%$ and $7.9 \%$ increases in the placebo group, respectively. A small French case-control study found no difference in bone mineral density and bone remodeling marker levels (serum C-terminal crosslinked type 1 telopeptide, procollagen type $1 \mathrm{~N}$-terminal propeptide, osteocalcin and bone-specific alkaline phosphatase) among chronic LBP patients with M1 or without MC [20].

In the chemokine panel, IP-10 was elevated at one month and at one year, while MCP-1 was elevated at one month in the ZA group. Both IP-10 and MCP-1 have been linked to pain [26-29]. In animal models, ZA reduced the expression of a number of pro-inflammatory and angiogenic mediators, including MCP-1 [30], whereas in our study, MCP-1 was elevated in the ZA group.

In the pro-inflammatory panel, IFN-A was elevated after one year in the ZA group-although not significantly so after B-H correction. Bisphosphonates do not only inhibit osteoclasts; they have also shown to suppress the secretion of proinflammatory cytokines such as IL-1, TNF-A, and IL-6 [31]. Several studies have found upregulation of pro-inflammatory cytokines in endplates with MC [32-34] and local anti-inflammatory treatment with glucocorticoid has shown to have a short-term alleviating effect on LBP [35]. In our RCT, the improvement in LBP in the ZA group [21] could have been due to the general ability of bisphosphonates to regulate bone turnover by suppressing osteoclast activity [36] or to direct anti-inflammatory effects.

In the vascular injury panel, hs-CRP and SAA were elevated after one month in the ZA group (but not significantly after B-H correction), but at one year there was no difference. Some studies have shown that administration of bisphosphonates elevates the levels of circulating CRP, IL-6, TNF-A, and cortisol levels [37,38]. ZA treatment often causes an acute phase reaction with fever and flu-like symptoms [39]. The published studies of inflammation parameters in MC are based on tissue samples [32,40,41], but only a few have assessed serum biomarkers. Rannou et al. found that high-sensitivity C-reactive protein (hsCRP) levels were elevated among patients with M1 and CLBP [19]. An Australian study demonstrated that elevation of CRP and IL- 6 during the acute phase of LBP was associated with better recovery, whereas individuals with elevated TNF had poorer prognoses [42]. It might be that the "window of opportunity" to detect inflammatory serum biomarkers only exists in the early phase of MC development and shortly after the infusion of bisphosphonates. 
A recent Dutch systematic review found moderate evidence of a positive association between the pro-inflammatory biomarkers CRP and IL- 6 and the severity of nonspecific LBP, and a positive association between TNF-A and the presence of nonspecific LBP [43]. If it were possible to determine a fingerprint in the serum of patients with LBP that is associated with MC, serum profiling could be used to assess the prognosis and choice of treatment. Khan et al. [44] emphasized the potential of diagnostic biomarkers to guide an era of individualized spine medicine for personalized therapeutics in the treatment of LBP. In our study, however, the changes in LBP intensity did not correlate with the changes in any biomarker's concentration.

Because of their potent antiresorptive activity, bisphosphonates, including ZA, represent standards of care for the treatment of osteoporosis. Studies in vitro and in vivo have shown that, for example, pamidronate and clodronate inhibit the synthesis of proinflammatory cytokines $[45,46]$. It has been proposed that the development of MC is dependent on the structural disruption of the intervertebral disc or endplate, and the inflammatory potential of the disc and the capacity of the bone marrow to respond to higher inflammatory stimuli [13]. The source of pain is probably the chemical and mechanical stimulation of the nociceptors adjacent to the damaged endplates. It has also been suggested that a low virulent infection of anaerobic bacteria may induce edema and inflammation [47]. When we exclude the theory of anaerobic infection from the pathophysiology of MC, we notice that ZA has several potential mechanisms for acting in MC lesions and is a promising, well-known and well-tolerated drug for treating LBP associated with MC. In the present study we could not prove the downregulation of serum biomarkers, including cytokines and growth factors, after ZA administration among LBP patients with MC. This might be due to our small sample size.

The strength of the present study is its randomized study design. Further strengths are its complete follow-up with no dropouts and $100 \%$ adherence, as medication was administered intravenously. All the analyses were performed using established, validated measurement assays in high-quality laboratories. Longitudinal data enable a better view of the possible inflammatory component in LBP with MC. However, we also acknowledge some limitations in our study. The small sample size of this pilot study might be inadequate to demonstrate significant changes in biomarkers, and multiple testing might increase the rate of false positive results. Thus, our results should be interpreted with caution. The possible presence of comorbidities and adverse lifestyles may affect the proinflammatory mediators observed in the systemic circulation of patients. For example, obesity is associated with increased serum levels of IL-6 and TNF-A [48], and smoking is characterized by elevated serum levels of, for example, TNF-A and IL-1, as well as other cytokines [49].

\section{Conclusions}

The present data demonstrated that a single intravenous infusion of $5 \mathrm{mg}$ ZA gave rise to different trends in serum biomarkers in the ZA and placebo groups, leading to significantly different regulations at one-year follow-up for one chemokine (IP-10), whereas two bone metabolism biomarkers (AFOS and iPINP) decreased in the ZA group. ZA treatment had an expected downregulating effect on bone turnover markers. This study found an association, a positive correlation, between a change in iPINP concentration and the change in total volume of MC and M1 volume. The changes in LBP intensity did not correlate with the changes in any biomarker's concentration.

This is the first RCT to investigate ZA in MC and to observe how it is reflected in serum biomarkers. The evaluation of these biomarkers expands our understanding of the mechanism of MC and LBP and may thereby guide us to new diagnostic and therapeutic methods. It adds to our knowledge of the effects of ZA on MC and on the biomarkers signaling this process. We conclude that serum biomarkers can be used to monitor the treatment effect of ZA among LBP patients with MC. Larger studies are required to demonstrate the findings of this pilot study.

Supplementary Materials: Supplementary materials can be found at www.mdpi.com/link. 
Author Contributions: Conceptualization, H.A., S.D., J.L., S.G., and J.K.; Methodology, J.R., M.-L.M., H.A., M.A., and S.G.; Software, M.H.; Validation, M.-L.M., J.R. and S.G.; Formal Analysis, M.H., S.G., K.K., and M.-L.M.; Investigation, J.J., O.T. and J.N.; Resources, E.K. and J.K.; Writing-Original Draft Preparation, K.K.; Writing-Review and Editing, K.K., J.K., J.L., S.D., D.S., S.G., M.A., J.R., M.-L.M., H.A., J.J., J.N. and O.T.; Visualization, M.H. and K.K.; Supervision, J.K. and E.K.; Project Administration, M.-L.M., S.G. and K.K.; Funding Acquisition, J.K., M.A., S.G. and E.K.

Funding: Novartis Pharma provided investigational medications for the study and supported the conduct of the trial (<USD 10 000). The study was supported by the Hansjörg Wyss Research Award (AOSpine). The funders played no role in the study design, data collection or analysis, decision to publish, or in the preparation of the manuscript.

Conflicts of Interest: The authors declare no conflict of interest.

\section{Abbreviations}

$\begin{array}{ll}\text { AFOS } & \text { alkaline phosphatase } \\ \text { bFGF } & \text { fibroblast growth factor beta } \\ \text { COMP } & \text { cartilage oligomeric matrix protein } \\ \text { CTX1 } & \text { C telopeptide of type I collagen } \\ \text { Flt } & \text { fms related tyrosine kinase (=VEGFR1) } \\ \text { hs-CRP } & \text { high-sensitive C-reactive protein } \\ \text { HGF } & \text { hepatocyte growth factor } \\ \text { ICAM } & \text { intercellular adhesion molecule } \\ \text { IL } & \text { interleukin } \\ \text { IFN } & \text { interferon } \\ \text { IP } & \text { interferon- } \gamma \text {-inducible protein } \\ \text { iPINP } & \text { intact procollagen I N-terminal propeptide } \\ \text { LBP } & \text { low back pain } \\ \text { MC } & \text { Modic change } \\ \text { M1 } & \text { type } 1 \text { Modic change } \\ \text { M2 } & \text { type } 2 \text { Modic change } \\ \text { MCP } & \text { monocyte chemotactic protein } \\ \text { MDC, } & \text { macrophage derived chemokine } \\ \text { MIG } & \text { monokine induced by gamma-interferon } \\ \text { MIP } & \text { macrophage inflammatory protein } \\ \text { MRI } & \text { magnetic resonance imaging } \\ \text { ODI } & \text { Oswestry Disability Index } \\ \text { RANKL } & \text { receptor activator of NF-kB ligand } \\ \text { RANTES } & \text { regulated upon activation, normally T-expressed, and presumably secreted } \\ \text { SAA } & \text { serum amyloid A } \\ \text { SD } & \text { standard deviation } \\ \text { TARC } & \text { thymus and activation-regulated chemokine } \\ \text { Tie } & \text { TEK receptor tyrosine kinase } \\ \text { TNF } & \text { tumor necrosis factor } \\ \text { VAS } & \text { Visual Analog Scale } \\ \text { VCAM } & \text { vascular cell adhesion molecule } \\ \text { VEGF } & \text { vascular endothelial growth factor } \\ \text { ZA } & \text { zoledronic acid } \\ & \end{array}$

\section{References}

1. De Roos, A.; Kressel, H.; Spritzer, C.; Dalinka, M. MR imaging of marrow changes adjacent to end plates in degenerative lumbar disk disease. Am. J. Roentgenol. 1987, 149, 531-534. [CrossRef] [PubMed]

2. Modic, M.T.; Steinberg, P.M.; Ross, J.S.; Masaryk, T.J.; Carter, J.R. Degenerative disk disease: Assessment of changes in vertebral body marrow with MR imaging. Radiology 1988, 166, 193-199. [CrossRef] [PubMed]

3. Modic, M.T.; Masaryk, T.J.; Ross, J.S.; Carter, J.R. Imaging of degenerative disk disease. Radiology 1988, 168, 177-186. [CrossRef] [PubMed] 
4. Perilli, E.; Parkinson, I.H.; Truong, L.H.; Chong, K.C.; Fazzalari, N.L.; Osti, O.L. Modic (endplate) changes in the lumbar spine: Bone micro-architecture and remodelling. Eur. Spine J. 2015, 24, 1926-1934. [CrossRef] [PubMed]

5. Kjaer, P.; Korsholm, L.; Bendix, T.; Sorensen, J.S.; Leboeuf-Yde, C. Modic changes and their associations with clinical findings. Eur. Spine J. 2006, 15, 1312-1319. [CrossRef]

6. Jensen, T.S.; Karppinen, J.; Sorensen, J.S.; Niinimaki, J.; Leboeuf-Yde, C. Vertebral endplate signal changes (Modic change): A systematic literature review of prevalence and association with non-specific low back pain. Eur. Spine J. 2008, 17, 407-422. [CrossRef]

7. Määttä, J.H.; Wadge, S.; MacGregor, A.; Karppinen, J.; Williams, F.M. ISSLS Prize Winner: Vertebral Endplate (Modic) Change is an Independent Risk Factor for Episodes of Severe and Disabling Low Back Pain. Spine (Phila Pa 1976) 2015, 40, 1187-1193. [CrossRef]

8. Toyone, T.; Takahashi, K.; Kitahara, H.; Yamagata, M.; Murakami, M.; Moriya, H. Vertebral bone-marrow changes in degenerative lumbar disc disease. An MRI study of 74 patients with low back pain. J. Bone Jt. Surg. Br. 1994, 76, 757-764. [CrossRef]

9. Kjaer, P.; Leboeuf-Yde, C.; Korsholm, L.; Sorensen, J.S.; Bendix, T. Magnetic resonance imaging and low back pain in adults: A diagnostic imaging study of 40-year-old men and women. Spine (Phila Pa 1976). 2005, 30, 1173-1180. [CrossRef]

10. Kuisma, M.; Karppinen, J.; Niinimäki, J.; Ojala, R.; Haapea, M.; Heliövaara, M.; Korpelainen, R.; Taimela, S.; Natri, A.; Tervonen, O. Modic changes in endplates of lumbar vertebral bodies: Prevalence and association with low back and sciatic pain among middle-aged male workers. Spine (Phila Pa 1976) 2007, 32, 1116-1122. [CrossRef]

11. Järvinen, J.; Karppinen, J.; Niinimäki, J.; Haapea, M.; Grönblad, M.; Luoma, K.; Rinne, E. Association between changes in lumbar Modic changes and low back symptoms over a two-year period. BMC Musculoskelet. Disord. 2015, 16, 98. [CrossRef] [PubMed]

12. Jensen, R.K.; Leboeuf-Yde, C.; Wedderkopp, N.; Sorensen, J.S.; Jensen, T.S.; Manniche, C. Is the development of Modic changes associated with clinical symptoms? A 14-month cohort study with MRI. Eur. Spine J. 2012, 21, 2271-2279. [CrossRef] [PubMed]

13. Dudli, S.; Fields, A.J.; Samartzis, D.; Karppinen, J.; Lotz, J.C. Pathobiology of Modic changes. Eur. Spine J. 2016, 25, 3723-3734. [CrossRef] [PubMed]

14. Dudli, S.; Miller, S.; Demir-Deviren, S.; Lotz, J.C. Inflammatory response of disc cells against Propionibacterium acnes depends on the presence of lumbar Modic changes. Eur. Spine J. 2018, 27, 1013-1020. [CrossRef]

15. Dudli, S.; Liebenberg, E.; Magnitsky, S.; Lu, B.; Lauricella, M.; Lotz, J.C. Modic type 1 change is an autoimmune response that requires a proinflammatory milieu provided by the "Modic disc". Spine J. 2018, 18, 831-844. [CrossRef]

16. Magnitsky, S.; Dudli, S.; Tang, X.; Kaur, J.; Diaz, J.; Miller, S.; Lotz, J.C. Quantification of Propionic Acid in the Bovine Spinal Disk After Infection of the Tissue With Propionibacteria acnes Bacteria. Spine (Phila Pa 1976) 2018, 43, E634-E638. [CrossRef]

17. Gornet, M.G.; Peacock, J.; Claude, J.; Schranck, F.W.; Copay, A.G.; Eastlack, R.K.; Benz, R.; Olshen, A.; Lotz, J.C. Magnetic resonance spectroscopy (MRS) can identify painful lumbar discs and may facilitate improved clinical outcomes of lumbar surgeries for discogenic pain. Eur. Spine J. 2019, 28, 674-687. [CrossRef]

18. Biomarkers Definitions Working Group. Biomarkers and surrogate endpoints: Preferred definitions and conceptual framework. Clin. Pharmacol. Ther. 2001, 69, 89-95. [CrossRef]

19. Rannou, F.; Ouanes, W.; Boutron, I.; Lovisi, B.; Fayad, F.; Mace, Y.; Borderie, D.; Guerini, H.; Poiraudeau, S.; Revel, M. High-sensitivity C-reactive protein in chronic low back pain with vertebral end-plate Modic signal changes. Arthritis Rheum. 2007, 57, 1311-1315. [CrossRef]

20. Teboul-Core, S.; Roux, C.; Borderie, D.; Kolta, S.; Lefevre-Colau, M.M.; Poiraudeau, S.; Rannou, F.; Nguyen, C. Bone mineral density and bone remodeling markers in chronic low back pain patients with active discopathy: A case-control exploratory study. PLoS ONE 2018, 13, e0196536. [CrossRef]

21. Koivisto, K.; Kyllönen, E.; Haapea, M.; Niinimäki, J.; Sundqvist, K.; Pehkonen, T.; Seitsalo, S.; Tervonen, O.; Karppinen, J. Efficacy of zoledronic acid for chronic low back pain associated with Modic changes in magnetic resonance imaging. BMC Musculoskelet. Disord. 2014, 15, 64. [CrossRef]

22. Fairbank, J.C.; Pynsent, P.B. The Oswestry Disability Index. Spine (Phila Pa 1976) 2000, 25, $2940-2952$. [CrossRef] 
23. Koivisto, K.; Järvinen, J.; Karppinen, J.; Haapea, M.; Paananen, M.; Kyllönen, E.; Tervonen, O.; Niinimäki, J. The effect of zoledronic acid on type and volume of Modic changes among patients with low back pain. BMC Musculoskelet. Disord. 2017, 18, 274. [CrossRef]

24. Black, D.M.; Reid, I.R.; Boonen, S.; Bucci-Rechtweg, C.; Cauley, J.A.; Cosman, F.; Cummings, S.R.; Hue, T.F.; Lippuner, K.; Lakatos, P.; et al. The effect of 3 versus 6 years of zoledronic acid treatment of osteoporosis: A randomized extension to the HORIZON-Pivotal Fracture Trial (PFT). J. Bone Miner. Res. 2012, 27, 243-254. [CrossRef]

25. Tan, W.; Sun, J.; Zhou, L.; Li, Y.; Wu, X. Randomized trial comparing efficacies of zoledronate and alendronate for improving bone mineral density and inhibiting bone remodelling in women with post-menopausal osteoporosis. J. Clin. Pharm. Ther. 2016, 41, 519-523. [CrossRef]

26. Al-Mazidi, S.; Farhat, K.; Nedjadi, T.; Chaudhary, A.; Zin Al-Abdin, O.; Rabah, D.; Al-Zoghaibi, M.; Djouhri, L. Association of Interleukin-6 and Other Cytokines with Self-Reported Pain in Prostate Cancer Patients Receiving Chemotherapy. Pain Med. 2017, 19, 1058-1066. [CrossRef]

27. Parkitny, L.; McAuley, J.H.; Di Pietro, F.; Stanton, T.R.; O'Connell, N.E.; Marinus van Hilten, J.J.; Moseley, GL. Inflammation in complex regional pain syndrome: A systematic review and meta-analysis. Neurology 2013, 80, 106-117. [CrossRef]

28. Romero-Sanchez, C.; Tsou, H.K.; Jan, M.S.; Wong, R.H.; Chang, I.C.; Londono, J.; Valle-Onate, R.; Howe, H.S.; $\mathrm{Yu}$, D.; Leung, B.P.; et al. Serum monocyte chemotactic protein-1 concentrations distinguish patients with ankylosing spondylitis from patients with mechanical low back pain. J. Spinal Disord. Tech. 2011, 24, $202-207$. [CrossRef]

29. Weber, K.T.; Satoh, S.; Alipui, D.O.; Virojanapa, J.; Levine, M.; Sison, C.; Quraishi, S.; Bloom, O.; Chahine, N.O. Exploratory study for identifying systemic biomarkers that correlate with pain response in patients with intervertebral disc disorders. Immunol. Res. 2015, 63, 170-180. [CrossRef]

30. Stathopoulos, G.T.; Moschos, C.; Loutrari, H.; Kollintza, A.; Psallidas, I.; Karabela, S.; Magkouta, S.; Zhou, Z.; Papiris, S.A.; Roussos, C.; et al. Zoledronic acid is effective against experimental malignant pleural effusion. Am. J. Respir. Crit. Care Med. 2008, 178, 50-59. [CrossRef]

31. Pennanen, N.; Lapinjoki, S.; Urtti. A.; Monkkonen, J. Effect of liposomal and free bisphosphonates on the IL-1 beta, IL-6 and TNF alpha secretion from RAW 264 cells in vitro. Pharm. Res. 1995, 12, 916-922. [CrossRef]

32. Ohtori, S.; Inoue, G.; Ito, T.; Koshi, T.; Ozawa, T.; Doya, H.; Saito, T.; Moriya, H.; Takahashi, K. Tumor necrosis factor-immunoreactive cells and PGP 9.5-immunoreactive nerve fibers in vertebral endplates of patients with discogenic low back Pain and Modic Type 1 or Type 2 changes on MRI. Spine (Phila Pa 1976) 2006, 31, 1026-1031. [CrossRef]

33. Fields, A.J.; Liebenberg, E.C.; Lotz, J.C. Innervation of pathologies in the lumbar vertebral end plate and intervertebral disc. Spine J. 2014, 14, 513-521. [CrossRef]

34. Chen, S.; Huang, Y.; Zhou, Z.J.; Hu, Z.J.; Wang, J.Y.; Xu, W.B.; Fang, X.Q.; Fan, S.W. Upregulation of tumor necrosis factor alpha and ADAMTS-5, but not ADAMTS-4, in human intervertebral cartilage endplate with modic changes. Spine (Phila Pa 1976) 2014, 39, E817-E825. [CrossRef]

35. Nguyen, C.; Boutron, I.; Baron, G.; Sanchez, K.; Palazzo, C.; Benchimol, R.; Paris, G.; James-Belin, E.; Lefevre-Colau, M.M.; Beaudreuil, J.; et al. Intradiscal Glucocorticoid Injection for Patients With Chronic Low Back Pain Associated With Active Discopathy: A Randomized Trial. Ann. Intern. Med. 2017, 166, 547-556. [CrossRef]

36. Brown, J.P.; Kendler, D.L.; McClung, M.R.; Emkey, R.D.; Adachi, J.D.; Bolognese, M.A.; Li, Z.; Balske, A.; Lindsay, R. The efficacy and tolerability of risedronate once a week for the treatment of postmenopausal osteoporosis. Calcif. Tissue Int. 2002, 71, 103-111. [CrossRef]

37. Sauty, A.; Pecherstorfer, M.; Zimmer-Roth, I.; Fioroni, P.; Juillerat, L.; Markert, M.; Ludwig, H.; Leuenberger, P.; Burckhardt, P.; Thiebaud, D. Interleukin-6 and tumor necrosis factor alpha levels after bisphosphonates treatment in vitro and in patients with malignancy. Bone 1996, 18, 133-139. [CrossRef]

38. Thiebaud, D.; Sauty, A.; Burckhardt, P.; Leuenberger, P.; Sitzler. L.; Green, J.R.; Kandra, A.; Zieschang, J.; Ibarra de Palacios, P. An in vitro and in vivo study of cytokines in the acute-phase response associated with bisphosphonates. Calcif. Tissue Int. 1997, 61, 382-392.

39. Al-Bogami, M.M.; Alkhorayef, M.A.; Bystrom, J.; Akanle, O.A.; Al-Adhoubi, N.K.; Jawad, A.S.; Mageed, R.A. Favorable therapeutic response of osteoporosis patients to treatment with intravenous zoledronate compared with oral alendronate. Saudi Med. J. 2015, 36, 1305-1311. [CrossRef] 
40. Torkki, M.; Majuri, M.L.; Wolff, H.; Koskelainen, T.; Haapea, M.; Niinimäki, J.; Alenius, H.; Lotz, J.; Karppinen, J. Osteoclast activators are elevated in intervertebral disks with Modic changes among patients operated for herniated nucleus pulposus. Eur. Spine J. 2016, 25, 207-216. [CrossRef]

41. Schroeder, G.D.; Markova, D.Z.; Koerner, J.D.; Rihn, J.A.; Hilibrand, A.S.; Vaccaro, A.R.; Anderson, D.G.; Kepler, C.K. Are Modic changes associated with intervertebral disc cytokine profiles? Spine J. 2017, 17, 129-134. [CrossRef]

42. Klyne, D.M.; Barbe, M.F.; van den Hoorn, W.; Hodges, P.W. ISSLS PRIZE IN CLINICAL SCIENCE 2018: Longitudinal analysis of inflammatory, psychological, and sleep-related factors following an acute low back pain episode-the good, the bad, and the ugly. Eur. Spine J. 2018, 27, 763-777. [CrossRef]

43. Van den Berg, R.; Jongbloed, E.M.; de Schepper, E.I.T.; Bierma-Zeinstra, S.M.A.; Koes, B.W.; Luijsterburg, P.A.J. The association between pro-inflammatory biomarkers and nonspecific low back pain: A systematic review. Spine J. 2018, 18, 2140-2151. [CrossRef]

44. Khan, A.N.; Jacobsen, H.E.; Khan, J.; Filippi, C.G.; Levine, M.; Lehman, R.A.; Riew, K.D.; Lenke, L.G.; Chahine, N.O. Inflammatory biomarkers of low back pain and disc degeneration: A review. Ann. N. Y. Acad. Sci. 2017, 1410, 68-84. [CrossRef]

45. Van Offel, J.F.; Schuerwegh, A.J.; Bridts, C.H.; Bracke, P.G.; Stevens, W.J.; De Clerck, L.S. Influence of cyclic intravenous pamidronate on proinflammatory monocytic cytokine profiles and bone density in rheumatoid arthritis treated with low dose prednisolone and methotrexate. Clin. Exp. Rheumatol. 2001, 19, 13-20.

46. Varenna, M.; Zucchi, F.; Ghiringhelli, D.; Binelli, L.; Bevilacqua, M.; Bettica, P.; Sinigaglia, L. Intravenous clodronate in the treatment of reflex sympathetic dystrophy syndrome. A randomized, double blind, placebo controlled study. J. Rheumatol. 2000, 27, 1477-1483.

47. Albert, H.B.; Lambert, P.; Rollason, J.; Sorensen, J.S.; Worthington, T.; Pedersen, M.B.; Norgaard, H.S.; Vernallis, A.; Busch, F.; Manniche, C.; et al. Does nuclear tissue infected with bacteria following disc herniations lead to Modic changes in the adjacent vertebrae? Eur. Spine J. 2013, 22, 690-696. [CrossRef]

48. Fantuzzi, G. Adipose tissue, adipokines, and inflammation. J. Allergy Clin. Immunol. 2005, 115, 911-920. [CrossRef]

49. Petrescu, F.; Voican, S.C.; Silosi, I. Tumor necrosis factor-alpha serum levels in healthy smokers and nonsmokers. Int. J. Chronic Obstruct. Pulm. Dis. 2010, 5, 217-222. 